\title{
Time-Series Photometry: CCDs vs. PMTs
}

\author{
T.J. Kreidl \\ Lowell Observatory, Flagstaff, Arizona 86001 U.S.A.
}

\begin{abstract}
With the ability to obtain simultaneous photometry of many objects, CCD time-series photometry is a potentially powerful method for obtaining data, even under non-photometric conditions. In particular, the ability to utilize one or more comparison stars on the same frame without the need to move the telescope to a different field makes for a higher duty cycle than conventional photoelectric photometry. In addition, the ability to determine the local sky in a variety of ways plus the ability to use more complex analysis techniques such as profile fitting and curves of growth permits a variety of analysis options. Some of the advantages of utilizing CCDs and the techniques used in time-series photometry of compact objects are discussed. With the flexibility of modern CCD control systems, possibilities for real-time or near real-time data analysis using readily available computer technology are stressed. Brief discussions of periodicity analysis considerations and other aspects of the data acquisition are presented.
\end{abstract}

\section{Introduction and history}

Time-series photometry is basically an extension of classical differential photometry, except that with computer-controlled data acquisition systems and telescope control, it has become possible to work considerably more efficiently. The evolution from a two- or multi-star photometer to a CCD as a multi-object photometer was a natural consequence. It did, however, require that CCDs and their control systems reach a certain maturity in terms of achievable precision as well as adequate field coverage, not to mention reasonable readout times and adequate data storage space.

Some of the first such experiments were carried out by Howell and Jacoby (1986). An on-line system was described by Stover and Allen (1987). As soon as computers and disk storage became reasonably inexpensive, the use of CCDs for time-series photometry exploded. Once CCD systems came down in price and became more commercially available, their use on on small telescopes became possible even for observatories with modest resources. Before discussing some of the characteristics of CCDs, a brief review of photoelectric photometry (PP) is appropriate.

\section{Photoelectric photometry}

For PP of a single star, "bright" sources are dominated by scintillation noise and by sky measurement errors due to the need to use large apertures. Changes in seeing, 
periodic drive errors, or poor repetition of centering can have drastic consequences for small apertures. Precision is not limited by shot and scintillation noise. In general, the inability to see (and hence center on) very faint objects is itself a problem, and in addition, faint stars that can contaminate the aperture are often invisible or nearly so.

For differential photometry (DP), better results than for PP can be obtained in some cases. In particular, for studies of variability on time scales roughly greater than 20 minutes, DP is preferable. Problems can result from drifts in transparency on time scales similar to the sampling rate, and from poor centering or repeatability of aperture placement on the sky. Also, if large differences between star brightnesses exist, the poorer photon statistics of the fainter object degrade the end product. Finally, even with modern systems, there is an inherent loss of duty cycle switching between variable, comparison star(s) and sky measurements.

\section{CCDs and some of their characteristics}

CCDs aren't the panacea for photometrists, either, but do have some characteristics that make them very nice tools for time-series photometry. For CCDs, scintillation noise usually dominates for shorter exposures, but since one often wants to study faint objects, getting high total counts is more of a concern; exposure times tend to be minutes in length. The higher quantum efficiency of CCDs plus their excellent linearity make them almost ideal detectors. Since they can act as a multi-star photometer and obtain many objects on the same detector, the transparency is essentially identical for all the objects in the field (which is typically around a few arcminutes in diameter) and thus the observations are independent of transparency variations caused by imperfect observing conditions (even fairly thick clouds). For frequencies below about $1 \mathrm{mHz}$, a CCD is generally better than a PMT for this reason.

So, why, one may ask, do observers even bother using PP any more if the CCD has so many advantages? Let us turn now to some of the problems, since the real world is anything but ideal. For one, CCDs suffer from flat fielding errors; this may be one of the biggest error sources, since the characterization of a CCD is a timeconsuming task and its characteristics may not all remain stable over long periods of time. In addition, we are taught that we should take flats every night as there can be instrumental effects that differ night-to-night. Given the fact that each pixel has an associated error with it and the two-dimensional flux distribution not only moves around, but in addition, is not evenly distributed over the same pixels for every exposure, the problem becomes more obvious. Imperfect guiding and changes in seeing cause such an effect. There are also inherent hardware error sources in CCDs, such as charge transfer efficiency (due to the CCD itself, or to improper electronic settings that affect the readout process), cosmetic defects, hot pixels, cosmic ray events, and hysteresis (in some older CCDs). A/D converters can also be sources of problems: some suffer from linearity problems at extreme levels, have "sticky" bits, and can change characteristics with age. Not only is the CCD chip itself a source of errors, but so are its electronics settings and how the observer uses the system. For 
example, if the gain exceeds the readout noise, one undersamples the read noise and introduces additional errors; see e.g. Massey and Jacoby (1992) for a discussion of this and other related problems.

Still, it is possible to achieve rather good results, and above all, for certain types of projects, precision can be achieved that cannot be attained from ground-based telescopes with PP. Fig. 1 shows the theoretical S/N that can be achieved for a CCD vs. a PMT (assuming unrealistically that the PMT has the same quantum efficiency as the CCD and that no overhead exists for either system). Such an algorithm is easy to construct and valuable for estimating what to expect before observing. The expected signal-to-noise (S/N) ratio for PMT and CCD data can be readily predicted, and is discussed for PP in Henden and Kaitchuck (1990), and for CCDs in Howell (1992) and in Kjeldsen and Frandsen (1992).

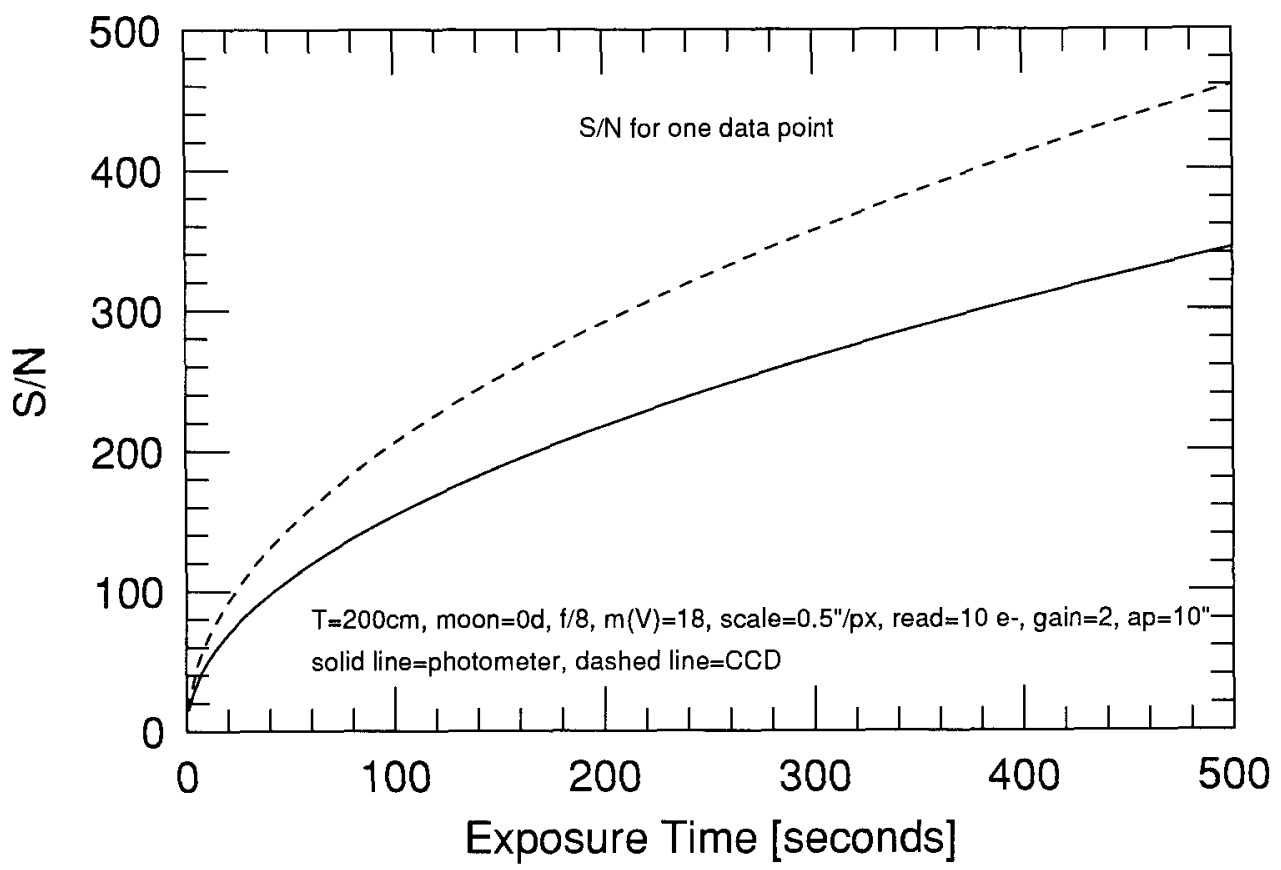

Figure 1 Signal-to-noise ratio compared for a single measurement with a CCD and a PMT. Note the unrealistic assumption that the $Q E$ is the same for both detectors. It is also assumed that a sky measurement of equal length was obtained independently with the PMT system. Here, the CCD sky determination used $10 \times$ the number of pixels as used to integrate the brightness of the star.

Clearly, the CCD beats the PMT for all but the very brightest objects; the use of sufficiently many CCD pixels for sky determination is important. Fig. 2 illustrates a typical data set obtained over nearly six hours; these particular data were reduced via IRAF's apphot. Fig. 3 shows the results of a Fourier analysis of data taken with 
different CCD chips of constant stars of quite different magnitudes. Using 300-second integrations, we have even managed to get reasonable light curves of stars as faint as $20 \mathrm{mag}$ in the red with a 1.8-m telescope (Howell et al. 1990).

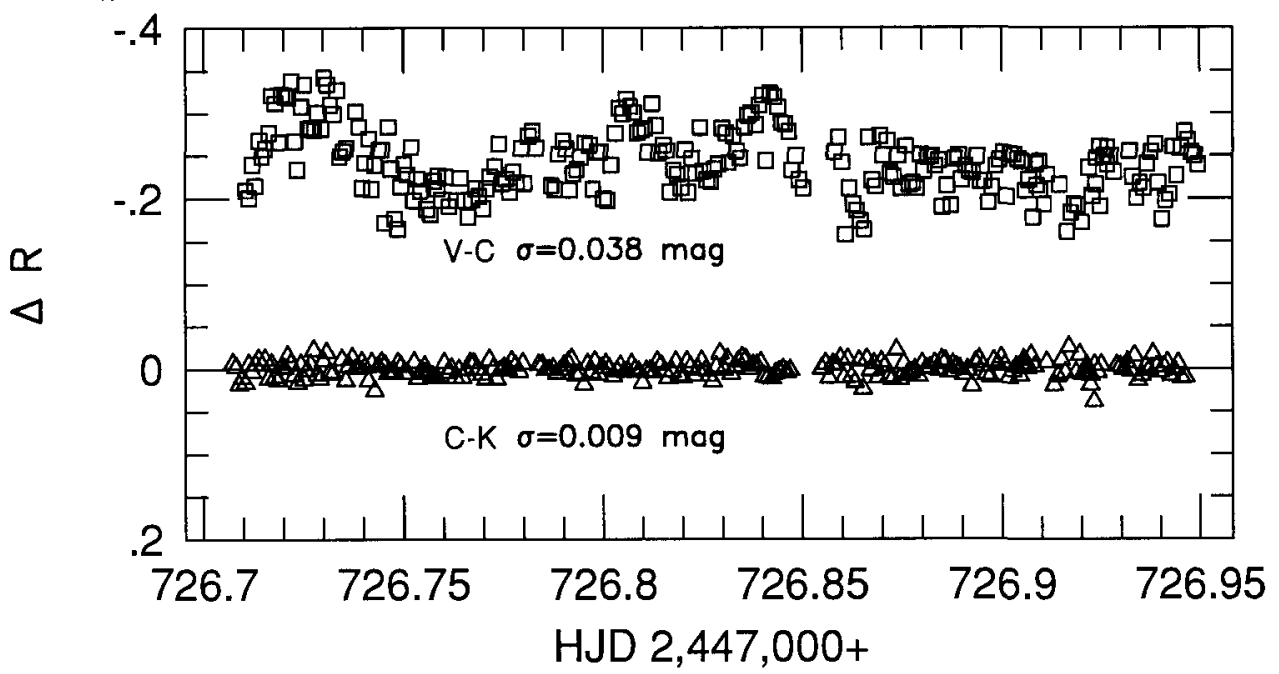

Figure 2 Differential CCD magnitudes of the cataclysmic variable V404 Cyg (V) and two comparison stars ( $C$ and $K$ ). Note the degree of activity of $\mathrm{V}-\mathrm{C}$ and the constancy of $\mathrm{C}-\mathrm{K}$.

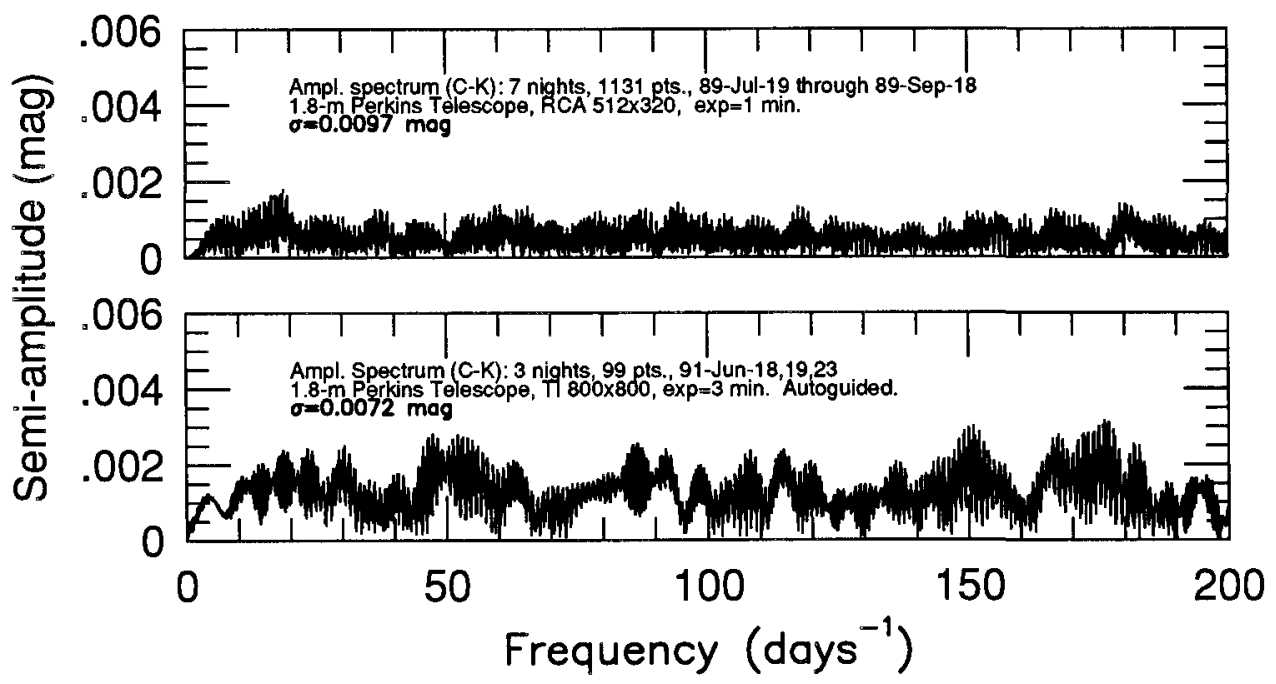

Figure 3 Amplitude spectra of two different, combined time series of comparison stars. $\mathrm{C}$ and $\mathrm{K}$ in the lower panel are about $3 \mathrm{mag}$ fainter than $\mathrm{C}$ and $\mathrm{K}$ in the upper panel. The noise level in the amplitude spectrum depends not only on the $\sigma$ of the data, but the number and spacing of the data and the period of time over which they were obtained. 


\section{Considerations for data acquisition and reduction}

Understanding the characteristics of the CCD itself is important both for data taking and reduction. The gain, range of linearity, readout noise, uniformity of response from pixel-to-pixel, stability, bias pattern, dark current buildup, and image scale (pixel oversampling rate) need to be considered.

A number of steps can be undertaken to improve upon simple aperture photometry. The use of comparison stars of similar brightness and color as the variable star is important; a quick look using a filter of different bandpass can be used to pick good comparison star candidates. The integration times or the filter width can be optimized (see Kjeldsen and Frandsen 1992). One must be careful not to undersample the expected period of variability. For a sinusoidal variation, the amplitude "suffers" as a function of $\sin c(\nu t)$ where $\nu$ is the frequency of the period and $t$ the data spacing (cf. Martinez 1989). For 1\% precision in one period, 14 points/cycle are needed, and at the Nyquist frequency ( 2 points/cycle), the amplitude is sampled only at the .637 level. The most important point I'd like to make is that the algorithm used for extracting magnitudes is critical. Simple aperture photometry generally will not do as good of a job as a more sophisticated algorithm, such as DAOPHOT (Stetson 1987), ROMAPHOT (Buonanno et al. 1983), DoPHOT (Mateo and Schechter 1989), MOMF (Kjeldsen and Frandsen 1992), or aperture correction methods (e.g., DaCosta et al. 1982; Howell 1989). It can be argued that, for non-crowded fields, this can be "overkill" to some extent, but there are potentially large differences in precision at stake. Clearly, for uncrowded or semi-crowded fields, the astronomical community needs some easy-to-use routines that improve on the simplistic approach of aperture photometry. MOMF seems to be a step in the right direction. It is equally as important to make sure users understand the algorithms utilized and don't just use programs as "black boxes." There are clearly many such routines in existence in the astronomical community, but few have been published.

In this regard, it should be noted that some reduction software packages assume pure Gaussian noise for the background, which for very low sky levels is not always the case (cf. Newberry 1992). Note also that a common estimate of the mode as $3 \times$ median $-2 \times$ mean is not always close to being correct.

Mention should be made of the ensemble photometry method (Gilliland and Brown 1992). This offers the most precise method available for searching for periodicities with amplitudes of a few tens of micromagnitudes in long time-series data, provided that sufficient numbers of high $\mathrm{S} / \mathrm{N}$ stars are present in the CCD frame.

This brings us to the topic of analysis. Periodicity analysis is a major subject in itself. Planning of the observations ( $\mathrm{S} / \mathrm{N}$, sample rate, gaps in the data, etc.) will affect the analysis one way or the other, and hence should be done carefully. The use of Fourier analysis, PDM or other periodicity routines should be followed up with an investigation of the window function, the significance of detection (see, e.g. Scargle 1982; Horne and Baliunas 1986; Stellingwerf 1978) as well as tests for variability, such 
as Howell et al. (1988).

\section{Future CCD systems}

The future holds much promise for CCD time-series photometry. We will see less pixel-to-pixel sensitivity variation (perhaps eventually obviating the need to flatfield). Some chips are already constant to the 1-to- $2 \%$ level (see the paper by A. Walker in this volume). Many CCD characteristics, such as quantum efficiency and linearity, are already nearly at theoretical levels and many newer detectors have substantial full well capacities and low readout noise, hence the need for better A/D conversion (20-24 bits and greater stability). Faster readout rates while maintaining good CTE would also be beneficial for time-series work. The ability to do a partial frame readout, or make use of multiple simultaneous readouts or the frame transfer mode available on some chips, can also save substantial time. Having powerful computers to do some analysis at the telescope can be very valuable; decisions can be made on whether or not to change observing strategies, keep taking data on an interesting object, etc. Even if a more precise analysis is performed later, some of us have found quick feedback to be extremely useful.

Finally, one ought to consider other means to get the information desired. For example, some stars can best be studied via RV variations where the characteristics of the CCD come in with a different weight than when used as a "pure" photometer.

I would like to thank S. B. Howell, R. M. Wagner, C. A. Gullixson, J. A. Holtzman, G. W. Lockwood, and countless other colleagues for many stimulating discussions.

\section{References:}

Buonanno, R. et al., 1983, Astron. Astrophys. 243, 160.

DaCosta, G.S., Ortolani, S., Mould, J., 1982, Ap. J. 257, 633.

Gilliland, R.L., Brown, T.M., 1992, Pub. Astron. Soc. Pacific 104, 582.

Henden, A.H., Kaitchuck, R.H, 1990, Astronomical Photometry, William-Bell, Inc., Richmond, Virginia.

Horne, J.H., Baliunas, S.L., 1986, Ap. J. 302, 757.

Howell, S.B., 1989, Pub. Astron. Soc. Pacific 101, 616.

Howell, S.B., 1992, in: A.S.P. Conf. Series, Vol. 23, p. 240.

Howell, S.B., Jacoby, G.H., 1986, Pub. Astron. Soc. Pacific 98, 802.

Howell, S.B., Mitchell, K.J., Warnock III, A., 1988, Astron. J. 95, 247.

Howell, S.B., Szkody, P., Kreidl, T.J., Mason, K.O., Puchnarewicz, E.M., 1990, Pub. Astron. Soc. Pacific 102, 758.

Kjeldsen, H., Frandsen, S., 1992, Pub. Astron. Soc. Pacific 104, 413.

Massey, P., Jacoby, G.H, 1992, in: A.S.P. Conf. Series, Vol. 23, p. 240.

Mateo, M., Schechter, P.L., 1989, in: Proc. First ESO/STECF Data Analysis Workshop, ESO, Garching, p. 69.

Martinez, P., 1989, Mon. Not. R. Astron. Soc. 248, 439.

Newberry, M.V., 1992, A.S.P. Conf. Series, Vol. 25, p. 307.

Scargle, J.D., 1982, Ap. J 263, 835.

Stellingwerf, R.F., 1978, Ap. J. 224, 953.

Stetson, P.B., 1987, Pub. Astron. Soc. Pacific 99, 191.

Stover, R.J., Allen, S.L., 1987, Pub. Astron. Soc. Pacific 99, 877. 


\section{Discussion}

D. O'Donoghue: Did I understand you to say that the $S / N$ ratio is always better for CCD's rather than photomultipliers, even for bright stars.

Kreidl: For very bright stars, photon statistics dominate the error, and precision should be essentially the same. The important point is that for time series, CCDs eliminate transparency variations, so, in particular for variability at frequencies below one or two millihertz, one can often do better with a CCD. This assumes that other sources of error can be adequately minimized, of course (which can be difficult).

For faint stars, the determination of the sky background increasingly becomes extremely important, so the CCD will always have the potential to do better than a photoelectric photometer. 